\title{
Bounds on Completely Convergent Euclidean Feynman Graphs
}

\author{
J. Feldman *, J. Magnen, V. Rivasseau, and R. Sénéor \\ Centre de Physique Théorique de l'Ecole Polytechnique, Plateau de Palaiseau, \\ F-91128 Palaiseau, Cedex, France
}

\begin{abstract}
Let $G$ be a Euclidean Feynman graph containing $L(G)$ lines. We prove that if $G$ has massive propagators and does not contain any divergent subgraphs its value is bounded by $K^{L(G)}$. We also prove the infrared analogue of this bound.
\end{abstract}

\section{Introduction}

This paper gives a very flexible technique for bounding Euclidean Feynman graphs. Such bounds are crucial not only in directly perturbation theoretic results [dCR, $\mathrm{R}$, ' $\mathrm{tH}]$ but more generally in the proofs of convergence of many expansions in quantum field theory. (In fact, it was the demands of [FMRS 1] that led us to this work.) Typically, you need to estimate the rate of growth of the value of graphs as a function of their size, say as measured by the number of lines in the graph. In superrenormalizable models one can prove relatively easily $[\mathrm{G} 1, \mathrm{RS}]$ that

$$
|G| \leqq K^{L(G)}
$$

where $|G|$ is the value of the graph $G, K$ is a constant, and $L(G)$ is the number of lines in $G$. However, in strictly renormalizable models the bound (1.1) is no longer true. Explicit families of graphs with factorial growth have been constructed [L, $\mathrm{dCR}$. These factorials arise from the renormalization subtractions. In [dCR] it is proven that, in $\phi_{4}^{4}$, graphs that do not contain divergent subgraphs obey the bound (1.1). 't Hooft ['tH] has proven a similar result for planar $\phi_{4}^{4}$.

In this paper we generalize this result to pretty well any model. In Sect. II we consider the ultraviolet case. In Sect. III we consider the infrared case. This case is also covered by [dCPR]. In the appendix we consider the case of only "logarithmically" convergent graphs.

We use a phase space expansion. In fact, because there is not very much obscuring detail it is one of the simplest applications of a phase space expansion we

* Permanent address: Department of Mathematics, University of British Columbia, Vancouver B.C., Canada V6T 1 Y4 
know of. Nonetheless it is a powerful expansion. In a separate publication [FMRS 3] we modify the basic expansion of this paper to yield bounds on renormalized graphs.

In the course of completing this work we received preprints [GN, G 2] in which perturbative estimates are also derived from a phase space analysis, using a new and powerful formalism. Although this formalism, in contrast with this paper and [FMRS 3], is not aimed at the study of individual, rather general, Feynman graphs, the estimating techniques in [GN, G2] are very close to ours.

\section{The Ultraviolet Case}

In this section we prove a bound of the form (1.1) on all connected graphs $G$ obeying the hypotheses

\section{(H2.1) The Lines}

The $l^{\text {th }}$ line has a propagator which in (Euclidean) space-time is bounded by $K^{\prime} \int \frac{e^{i k \cdot(x-y)}}{\left(k^{2}+m^{2}\right)^{p(l)}} d^{d} k$ with $m>0, K^{\prime}$ independent of $l$ and $p(l)>0$. Matrix valued propagators (such as fermion propagators) are of course allowed.

\section{(H2.2) The Vertices}

Any number of lines may be hooked to any vertex.

\section{(H2.3) The External Vertices}

We view our graph $G$ as not containing any external lines. On the other hand, any vertex of $G$ may be designated an external vertex. The set of external vertices of $G$ must be nonempty and is denoted $\mathscr{V}_{E}(G)$. We provide estimates for three different treatments of the external vertices. (Internal vertices are integrated over all $\mathbb{R}^{d}$.)

(H2.3a)

Each $v \in \mathscr{V}_{E}(G)$ is integrated over a standard unit cube (i.e. over $n_{i, v} \leqq x_{i, v} \leqq n_{i, v}+1$ with $n_{i, v} \in \mathbb{Z}, 1 \leqq i \leqq d$ ) in position space $\mathbb{R}^{d}$.

( $H 2.3 b)$

Each $v \in \mathscr{V}_{E}(G)$ is integrated against a test function $f_{v}$ on position space $\mathbb{R}^{d}$.

(H2.3c)

Each $v \in \mathscr{V}_{E}(G)$ has a fixed external momentum $k_{v}$ entering it. In this case we also assume that the propagators are translation invariant.

\section{(H2.4) Superficial Convergence}

This is, of course, the crucial hypothesis. By a subgraph $G^{\prime} \cong G$ we mean simply a subset of the lines of $G$. The vertices of $G^{\prime}$ are the end points of lines of $G^{\prime}$ and an external line of $G^{\prime}$ is a line of $G \backslash G^{\prime}$ which is hooked to a vertex of $G^{\prime}$. We define 
$\Lambda\left(G^{\prime}\right)=$ \# loops of $G^{\prime}, E\left(G^{\prime}\right)=$ \# external lines of $G^{\prime}, L\left(G^{\prime}\right)=$ \# internal lines of $G^{\prime}$, where each external vertex of $G$ counts as having one line external to $G$. We assume that there is an $\varepsilon>0$ such that the superficial degree of divergence

$$
D\left(G^{\prime}\right) \equiv \Lambda\left(G^{\prime}\right) d-\sum_{l \in G^{\prime}} 2 p(l)
$$

of each connected subgraph of $G$ obeys

$$
-D\left(G^{\prime}\right) \geqq 2 \varepsilon E\left(G^{\prime}\right) .
$$

This is true in any renormalizable theory. For example in $\phi_{4}^{4}-D\left(G^{\prime}\right)=E\left(G^{\prime}\right)-4$ so that $-D\left(G^{\prime}\right) \geqq \frac{1}{5} E\left(G^{\prime}\right)$ for any connected $G^{\prime}$ which has $E\left(G^{\prime}\right) \geqq 5$, i.e. is convergent.

The value of the graph (which is also denoted $G$ ) is defined to be $\int \prod_{v} d x_{v} \prod_{l \in G} c_{l}\left(x_{l}, x_{l}\right)$, where $c_{l}$ is the propagator for the line $l$ and $x_{l}, x_{l}^{\prime}$ are the positions of the vertices at the ends of $l$.

Theorem 2.1. If for some $m, \varepsilon>0$ hypotheses (H2.1)-(H2.4) above are satisfied then for any $0<\zeta<1$ there exists a constant $K$ such that

$$
|G| \leqq K^{L(G)} \times \begin{cases}\sup _{x_{v}} \prod_{l \in G} e^{-m(1-\zeta)\left|x_{l}-y_{l}\right|} & \text { for }(\mathrm{H} 2.3 \mathrm{a}), \\ \inf _{v \in \mathscr{V}_{E}}\left\|f_{v}\right\|_{L^{1}} \prod_{v \neq w \in \mathscr{V}_{E}}\left\|f_{v}\right\|_{L^{\infty}} & \text { for }(\mathrm{H} 2.3 \mathrm{~b}), \\ \delta\left(\sum_{v \in \mathscr{V}_{E}} k_{v}\right) & \text { for }(\mathrm{H} 2.3 \mathrm{c}) .\end{cases}
$$

Here the sup runs over all possible positions of the vertices of $G$ but with the external vertices in the cubes of $(\mathrm{H} 2.3 \mathrm{a})$.

Remark 1 . The constant $K$ depends only on $m, \varepsilon, \zeta, d, K^{\prime}$, and the $p(l)$ 's.

Remark 2. We shall prove this theorem using a phase space expansion. Consequently, we must be able to localize simultaneously in "momentum space and position space." To do so we simply use Lemma 2.2 below to bound each propagator in $G$. The right-hand side of the bound (2.3) should be thought of as resulting from a decomposition of momentum space into slices indexed by $i$ with the momentum in slice $i$ being of magnitude roughly $M^{i}$. For notational convenience we have scaled the mass $m$ to 1 both in Lemma 2.2 and in the proof of Theorem 2.1.

Remark 3. We will use $K$ to denote many different (and largely irrelevant) constants.

Lemma 2.2. For any $p>0, \zeta>0$, and $M>1$ there exists a constant $K=K(\zeta, M, d, p)$ such that

$$
|C(x, y)| \equiv\left|K^{\prime} \int \frac{d^{d} k}{(2 \pi)^{d}} \frac{e^{i k \cdot(x-y)}}{\left(k^{2}+1\right)^{p}}\right| \leqq K \sum_{i=0}^{\infty} M^{i(d-2 p)} e^{-(1-\zeta / 2) M^{i}|x-y|} .
$$

Proof. Taking the Fourier transform of

$$
\frac{1}{\left(k^{2}+1\right)^{p}}=\frac{1}{\Gamma(p)} \int_{0}^{\infty} d \alpha \alpha^{p-1} e^{-\alpha\left(k^{2}+1\right)}, \quad p>0,
$$


gives the $\alpha$-representation

$$
C(x, y)=\frac{1}{(4 \pi)^{d / 2}} \frac{K^{\prime}}{\Gamma(p)} \int_{0}^{\infty} d \alpha \alpha^{p-d / 2-1} e^{-\alpha} e^{-\frac{|x-y|^{2}}{4 \alpha}}
$$

from which follows the upper bound

$$
|C(x, y)| \leqq K e^{-\left(1-\frac{5}{3}\right)|x-y|} \begin{cases}\frac{1}{|x-y|^{d-2 p}} & \text { if } \quad d-2 p>0 \\ 1+|\ln | x-y|| & \text { if } d-2 p=0 \\ 1 & \text { if } d-2 p<0 .\end{cases}
$$

Hence it suffices to prove the corresponding lower bound on the right-hand side of (2.3). Now the bound

$$
\sum_{i=0}^{\infty} M^{i(d-2 p)} e^{-(1-\zeta / 2) M^{i}|x-y|} \geqq M^{O(d-2 p)} e^{-(1-\zeta / 2) M^{0}|x-y|}=e^{-(1-\zeta / 2)|x-y|}
$$

is adequate for $|x-y| \geqq 1$ and for $d-2 p<0,|x-y| \leqq 1$. For $|x-y| \leqq 1, d-2 p \geqq 0$ we use

$$
\int_{1}^{\infty} d s s^{\delta-1} e^{-s r}=\sum_{i=0}^{\infty} \int_{M^{i}}^{M^{i+1}} d s \frac{s^{\delta}}{S} e^{-s r} \leqq \sum_{i=0}^{\infty}\left(M^{i+1}-M^{i}\right) \frac{M^{\delta(i+1)}}{M^{i}} e^{-M^{i r}}
$$

to yield

$$
\begin{aligned}
& \sum_{i=0}^{\infty} M^{i(d-2 p)} e^{-(1-\zeta / 2) M^{i}|x-y|} \\
& \geqq \frac{1}{M^{\delta+1}} \int_{1}^{\infty} d s s^{d-2 p-1} e^{-(1-\zeta / 2)|x-y| s} \\
& \quad=K \frac{1}{|x-y|^{d-2 p}} \int_{|x-y|}^{\infty} d s s^{d-2 p-1} e^{-(1-\zeta / 2) s} .
\end{aligned}
$$

Finally, we observe that, for $|x-y| \leqq 1 d-2 p>0$

$$
\int_{|x-y|}^{\infty} d s s^{d-2 p-1} e^{-(1-\zeta / 2) s} \geqq \int_{1}^{\infty} d s s^{d-2 p-1} e^{-(1-\zeta / 2) s}>0
$$

and for $|x-y| \leqq 1, d-2 p=0$

$$
\int_{|x-y|}^{\infty} d s s^{-1} e^{-(1-\zeta / 2) s} \geqq K\left\{1+\int_{|x-y|}^{1} d s s^{-1}\right\}=K\{1+|\ln | x-y \|\} \cdot
$$

Proof of Theorem 2.1. We remind the reader that $m$ has been scaled to 1 . As well it suffices to prove the estimate associated with the hypothesis $(\mathrm{H} 2.3 \mathrm{a})$ since the other two estimates follow directly from it (using translation invariance).

For the reader's convenience we organize the proof into four steps: the preparation, the integral over position space, the harvesting of momentum space decay, and the sum over momentum space.

The Preparation. In this step we prepare the graph for application of the phase space expansion. First, we apply Lemma 2.2 (any $M>1$ well do) to bound the 
propagator associated with each line $l$ by

$$
\left|C_{l}(x, y)\right| \leqq K \sum_{i=0}^{\infty} M^{i \delta(l)} e^{-(1-\zeta / 2) M^{i}|x-y|}
$$

with $\delta(l)=d-2 p(l)$.

Pick a single term from the resulting (multi-) sum by assigning a single value of $i$ to each line. The sum over these "momentum assignments" will be performed in the third step. Next use

$$
M^{i}=1+\frac{M-1}{M} \sum_{j=1}^{i} M^{j}
$$

to rewrite the decay factor $e^{-(1-\zeta / 2) M^{i_{l}}|x-y|}$ associated with each line $l$ as

$$
\begin{aligned}
& e^{-(1-\zeta)|x-y|} e^{-\zeta / 2|x-y|} \prod_{j=1}^{i_{l}} e^{-(1-\zeta / 2) \frac{M-1}{M} M^{j}|x-y|} \\
& \leqq e^{-(1-\zeta)|x-y|} \prod_{j=0}^{i_{l}} e^{-\zeta^{\prime} M^{j}|x-y|}
\end{aligned}
$$

with $\zeta^{\prime}=\min \left\{\frac{\zeta}{2},(1-\zeta / 2) \frac{M-1}{M}\right\}$. The factors $e^{-(1-\zeta)|x-y|}$ are extracted to give the exponential decay between the external vertices quoted in the statement of the theorem. This leaves us with the problem of showing that the sum over momentum assignments of

$$
\int \prod_{v \neq v_{0}} d x_{v} \prod_{l \in G} M^{i_{l} \delta(l)} \prod_{j=0}^{i_{l}} e^{-\zeta^{\prime} M^{j}\left|x_{l}-y_{l}\right|}
$$

is bounded by $K^{L(G)}$. Here we have picked one external vertex $v_{0}$ at random and are holding it fixed. All other vertices (both internal and external) are integrated over all $\mathbb{R}^{d}$.

$\mathbb{R}^{d}$. following way. Define

$$
G_{j}=\left\{l \in G \mid i_{l} \geqq j\right\}
$$

and denote the set of connected components of $G_{j}$

$$
\begin{gathered}
\left\{G_{j}^{k} \mid 1 \leqq k \leqq q_{j}\right\} . \\
\text { Then } \prod_{l \in G} \prod_{j=0}^{i_{l}} e^{-\zeta^{\prime} M^{j}\left|x_{l}-y_{l}\right|}=\prod_{j, k} \prod_{l \in G_{j}^{k}} e^{-\zeta^{\prime} M^{j}\left|x_{l}-y_{l}\right|}
\end{gathered}
$$

with the product $\prod_{j, k}$ running over $j \geqq 0,1 \leqq k \leqq q_{j}$.

The integral over position space is based on the observation that given any set $v_{0}^{\prime}, v_{1}^{\prime}, \ldots, v_{n}^{\prime}$ of distinct vertices of $G_{j}^{k}$ we may reorder $v_{1}^{\prime}, \ldots, v_{n}^{\prime}$ [i.e. choose a permutation $\sigma$ of $(0,1, \ldots, n)$ with $\sigma(0)=0$ ] in such a way that

$$
\begin{aligned}
\int \prod_{i=1}^{n} d^{d} x_{v_{i}^{\prime}} \prod_{l \in G_{J}^{k}} e^{-\zeta^{\prime} M^{j}\left|x_{l}-y_{l}\right|} & \leqq \int \prod_{i=1}^{n} d^{d} x_{v_{l}^{\prime}} \prod_{i=1}^{n} e^{-\frac{1}{2} \zeta^{\prime} M^{j}\left|x_{v_{\sigma(i-1)}^{\prime}}-x_{v_{\sigma(i)}^{\prime}}\right|} \\
& =K^{n}\left(M^{-d j}\right)^{n}
\end{aligned}
$$

To see the first inequality throw away all the lines of $G_{j}^{k}$ except those forming some spanning tree $T_{j}^{k}$ of $G_{j}^{k}$. A spanning tree of $G_{j}^{k}$ is simply a subgraph of $G_{j}^{k}$ that has no 
Fig. 1

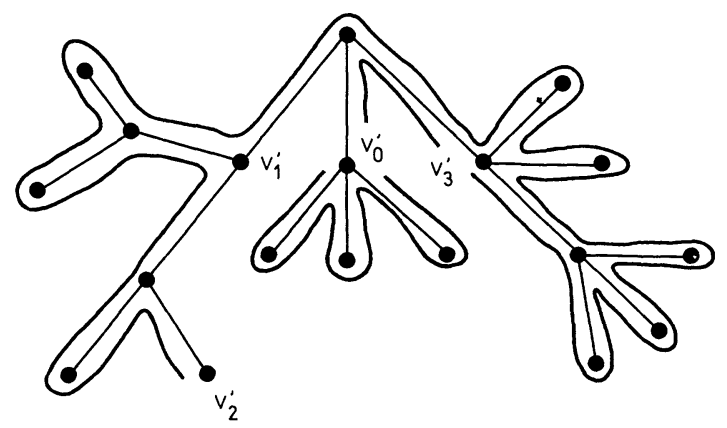

loops and which contains all the vertices of $G_{j}^{k}$. Then use the fact illustrated in Fig. 1 below that given any tree it is possible to find a path in the tree which starts at any given vertex (in our case $v_{0}^{\prime}$ ) passes through every vertex of the tree and which covers each line of the tree at most twice.

The bound on the integral over position space will be developed in the following way. We will below assign each vertex $v$ of $G$, except the fixed external vertex $v_{0}$, to a $G_{j}^{k}$ that contains $v$. The vertices assigned to $G_{j}^{k}$ will be called the integration vertices of $G_{j}^{k}$ and will play the role of $v_{1}^{\prime}, \ldots, v_{n}^{\prime}$ in (2.10). We will also pick for each $G_{j}^{k}$ a vertex to play the role of $v_{0}^{\prime}$. It will be called the fixed vertex of $G_{i}^{k}$. Then we apply

$$
\prod_{l \in G_{j}^{k}} e^{-\zeta^{\prime} M^{j}\left|x_{l}-y_{l}\right|} \leqq \prod_{i=1}^{n} e^{-\frac{1}{2} \zeta^{\prime} M^{j}\left|x_{v^{\prime} \sigma(i-1)}-x_{v_{\sigma(i)}^{\prime}}\right|}
$$

for any $j, k$. Finally, we integrate the positions of all integration vertices of each $G_{j}^{k}$ starting with the highest $j$ and considering successively smaller $j$ 's in turn. We demand that no integration vertex $v$ of $G_{j}^{k}$ be a fixed vertex of any $G_{j^{\prime}}^{k^{\prime}}$ with $j^{\prime}<j$. Consequently at the time $d x_{v}$ is performed the variable $x_{v}$ appears in only a single $e^{-\frac{1}{2} \zeta^{\prime} M^{j}\left|x_{v^{\prime}}-x_{v}\right|}$. Once we have done the integrations, as in (2.10), each vertex $v$ will be endowed with a factor $M^{-d j_{v}}$, where $j_{v}$ is the momentum scale of the $G_{j_{v}}^{k}$ to which $v$ has been assigned as an integration vertex. To make this factor as small as possible we would like $j_{v}$ as large as possible. To this end we also demand that every vertex of $G_{j}^{k}$ except the one fixed vertex of $G_{j}^{k}$ is an integration vertex of some $G_{j^{\prime}}^{k^{\prime}}$ with $j^{\prime} \geqq j$.

We now choose the fixed and integration vertices starting with the fixed vertices. Consider all $G_{j}^{k}$ 's. Assign $v_{0}$ as fixed vertex to all $G_{j}^{k}$ 's in which it appears and delete these $G_{j}^{k}$ 's from consideration. Pick at random any vertex $v_{0}^{\prime}$ of any remaining maximal (with the ordering given by containment) $G_{j}^{k}$. Assign this $v_{0}^{\prime}$ as fixed vertex to all remaining $G_{j}^{k}$ s in which it appears and delete these $G_{j}^{k}$ 's from consideration. Repeat until all $G_{j}^{k}$ s have been assigned fixed vertices. For each $v \neq v_{0}$ consider all $G_{j}^{k}$ s in which it appears. Assign it as an integration vertex to the $G_{j}^{k}$ with the largest $j$ for which it is not a fixed vertex.

\section{The Harvesting of Momentum Space Decay}

At the conclusion of the integral over position space we have the bound

$$
\int \prod_{v \neq v_{0}} d x_{v} \prod_{l \in G} M^{i_{l} \delta(l)} \prod_{j=0}^{i_{l}} e^{-\zeta^{\prime} M^{j}\left|x_{l}-y_{l}\right|} \leqq \prod_{l \in G} M^{i_{l} \delta(l)} \prod_{v \neq v_{0}}\left(K M^{-d j_{v}}\right),
$$


where $j_{v}$ is the momentum scale in which the integration over $x_{v}$ occurred. Now

$$
\begin{gathered}
M^{i_{l} \delta(l)}=\prod_{j=1}^{i_{l}} M^{\delta(l)}=\prod_{1 \leqq j \text { s.t. } l \in G_{j}} M^{\delta(l)}, \\
M^{-d j_{v}}=\prod_{j=1}^{j_{v}} M^{-d}=\prod_{\substack{1 \leq j \text { s.t. } v \in G_{j}, v \text { not fixed in scale } j}} M^{-d} .
\end{gathered}
$$

Hence, using $V(G)$ to denote the number of vertices of $G$,

$$
\begin{array}{rlrl}
\prod_{l \in G} M^{i \delta \delta(l)} \prod_{v \neq v_{0}}\left(K M^{-d j_{v}}\right) & =\prod_{j \geqq 1} \prod_{k} M^{-d\left(V\left(G_{j}^{k}\right)-1\right)+} \sum_{l \in G_{j}^{k}}^{\sum_{j} \delta(l)} \\
& \leqq K^{L(G)} \prod_{j \geqq 1} \prod_{k} M^{D\left(G_{j}^{k}\right)} & & \text { for a new } K, \\
& \leqq K^{L(G)} \prod_{j \geqq 1} \prod_{k} M^{-2 \varepsilon E\left(G_{j}^{k}\right)} & & \text { by }(\mathrm{H} 2.4)
\end{array}
$$

The factors of $M^{-2 \varepsilon}$ are reassigned as follows. Each $G_{j}^{k}$ is considered in turn starting with the highest $j$. For fixed $j, k$ each external vertex of $G_{j}^{k}$ is considered in turn. A factor of $M^{-\varepsilon}$ is assigned to each external line of $G_{j}^{k}$ ending at the external vertex in question. Given any line $l$ ending at any vertex $v$, there is a $k$ such that $l$ is an external line of $G_{j}^{k}$ with $v$ an external vertex of $G_{j}^{k}$ if and only if

$$
i_{l}<j \leqq \max \left\{i_{l}^{\prime} \mid l^{\prime} \text { ends at } v\right\} \equiv i_{v} .
$$

Consequently given any vertex $v$ and any line $l$ ending at $v$, the half of $l$ nearest $v$ is assigned

in total.

$$
M^{-\varepsilon\left(i_{v}-i_{l}\right)}
$$

\section{The Sum over Momentum Space}

To complete the proof of Theorem 2.1 we need only perform the sum over the different possible assignments of momentum scales to the lines of $G$. This sum will be controlled using the exponential decay between the momentum scales of lines meeting at a common vertex we have just produced. In principal the technique is identical to that used to bound a tree graph whose propagators have integrable decay in position space. In practice the construction of the appropriate dual tree will be a little messy. If, however, one is willing to allow a dependence on $v$, the maximum number of lines ending at a common vertex, in the final bound one can use

$$
\prod_{l \text { ending at } v} M^{-\varepsilon\left(i_{v}-i_{l}\right)} \leqq \prod_{l_{1}, l_{2} \text { ending at } v} M^{-\frac{\varepsilon}{v^{2}}\left|i_{l_{1}}-i_{l_{2}}\right|}
$$

to trivially provide a bound on the sum over momentum assignments [FMRS 2].

We start by dividing all possible momentum assignments into classes according to which line has the highest momentum at each vertex. Decide ties by numbering in some arbitrary way all lines in $G$ and using the rule "ties go to the line with the smallest number." Since there are

$$
\prod_{\text {vertices }}(\# \text { lines ending at the vertex }) \leqq \prod_{v} 2^{\# \text { lines ending at } v} \leqq 2^{2 L(G)},
$$

it suffices to consider momentum assignments consistent with any given fixed class. To maximize the similarity between the sum over momentum assignments and a 
classical sum over position space we will construct a tree graph $M$. To minimize confusion as we slowly build $M$ out of $G$ we will prefix all lines, vertices etc. of $M$ by $M$ - (as in momentum) and all lines, vertices etc. of $G$ by $G$-. The set of $M$-vertices will be precisely the set of $G$-lines. There will be associated with each $M$-line an exponential decay factor between the momentum scales of the $M$-vertices at its ends. (Hence we wish each $M$-line to join two $G$-lines which have a $G$-vertex in common, with one of the $G$-lines being the highest line of this $G$-vertex.) We construct $M$ in five steps.

Step 1. Start by drawing an $M$-vertex in the centre of each $G$-line. In the pictures below

- is a $G$-vertex,

- is a $G$-line,

$\curvearrowleft$ means that the line at the tail of the arrow is the highest line at the head of the arrow,

--- is an $M$-line,

$\bigcirc$ is an $M$-vertex.

Step 2. Now collapse any $G$-line which is simultaneously the highest line of two $G$-vertices into the $M$-vertex at its centre. Note that this $M$-vertex has absorbed exactly two $G$-vertices because, by construction, a $G$-vertex must have exactly one designated highest line. Hence in the picture below the $G$-lines without arrows $m u s t$ not be highest lines for two $G$-vertices at once.
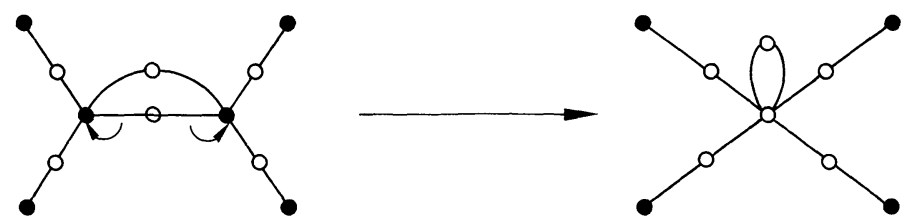

Step 3. There is now a one to one correspondence between the remaining $G$-vertices and the remaining highest $G$-lines. (That was the point of Step 2.) Replace each remaining $\_-$by o--- For example

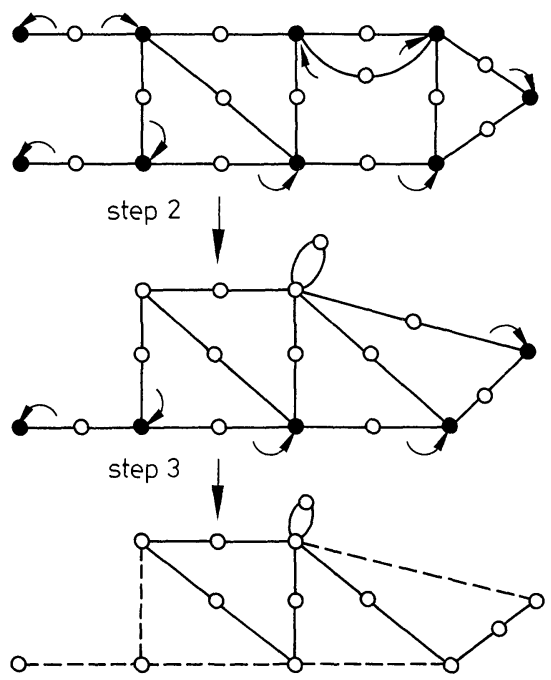


The situation is now the following.

- There are no $G$-vertices left although there may still be plenty of $G$-lines (each with an $M$-vertex drawn at its centre).

- Each $M$-vertex that is not drawn in the middle of a $G$-line ( $-\infty)$ corresponds to the highest line of some $G$-line.

- Each $M$-line connects two $M$-vertices (=G-lines) that had a $G$-vertex in common. To check this simply review the history that led to the introduction of a line $-L^{---}$.

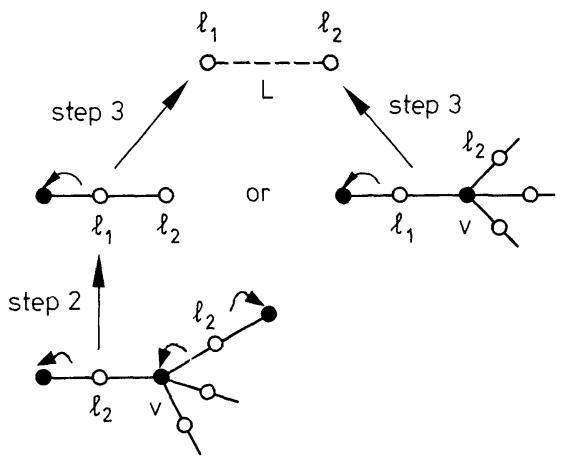

In both these cases $v$ is the $G$-vertex common to the $G$-lines $l_{1}$ and $l_{2}$. We also see that in both cases one of the $M$-vertices (in fact $l_{2}$ ) is the highest line of the common $G$-vertex, so we do indeed have the desired exponential decay factor associated with $L$.

- There are no closed loops made up solely of $M$-lines. For if there were $G$ must contain a closed loop of highest momentum lines. But that is impossible because the lines of $G$ are strictly ordered by momentum scale (thanks to the tie breaking mechanism) and each $G$-vertex must have exactly one highest momentum line.

Step 4. Repeat the following operation until no closed loops remain. Pick any

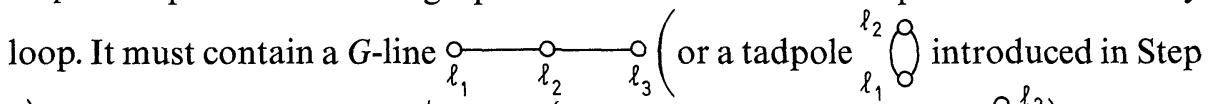

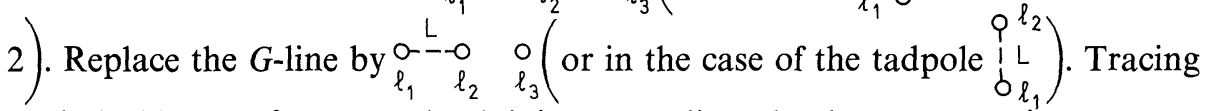
back the history of $L$ we see that it joins two $G$-lines that have a vertex in common, with one of the $G$-lines being the highest line of the common vertex.
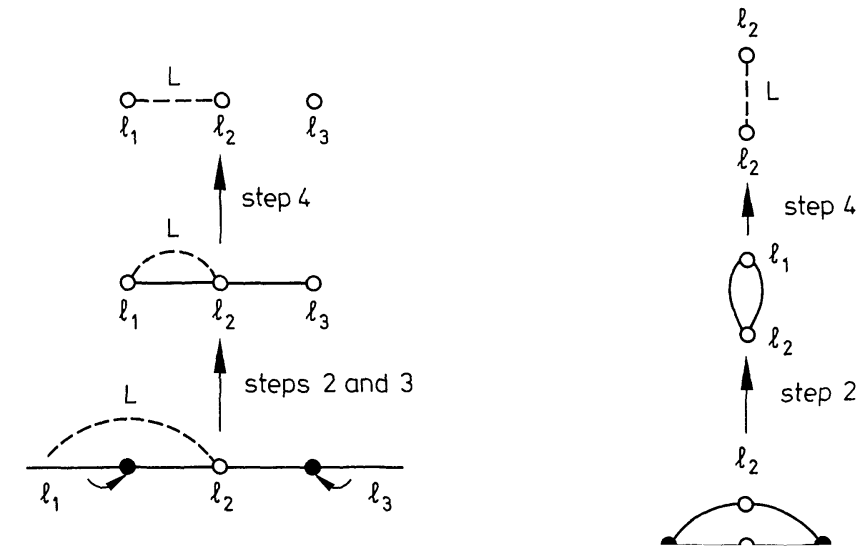


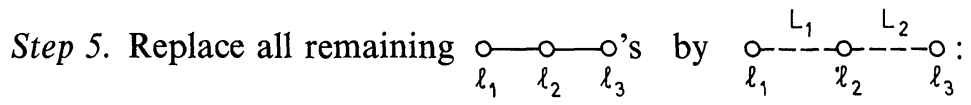

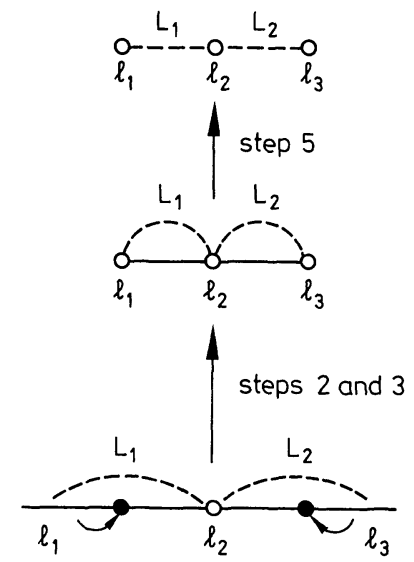

As usual we check that $L_{1}$ and $L_{2}$ have the desired decay factors.

We have now completed our construction of $M$. It is indeed a connected tree graph whose vertices are in one to one correspondence with lines of $G$ and whose lines are each endowed with a decay factor, $M^{-\varepsilon|i-j|}$, where $i$ and $j$ are the momentum scales of the $M$-vertices (=G-lines) at the ends of the $M$-line. Furthermore, for the purpose of this construction we can include in $G$ one external line for each external vertex of $G$ [see (H2.4)]. Since these (globally) external lines are not contained in any $G_{j}$ they accumulate factors of $M^{-\varepsilon}$ (in the harvest of decay in momentum space) as though they have momentum scale 0 . Consequently, the corresponding $M$-vertices have their momentum scales fixed at 0 . So we can perform the sum over momentum scales in the usual way, by introducing a natural partial ordering on the vertices of $M$ with one of the $i=0$ vertices being the "biggest" vertex and so on. The sum generates a constant (bounded by $\left.\sum_{i=-\infty}^{\infty} M^{-\varepsilon|i|}\right)$ per $G$-line.

\section{The Infrared Case}

We now move onto the infrared analogue of Theorem 2.1. As in Sect. II we take a position space point of view. We consider connected graphs consisting of external vertices, whose positions are fixed in (Euclidean) space-time, internal vertices whose positions are integrated over (Euclidean) space-time and lines joining pairs of distinct vertices. There are no external lines in the sense that one end of the line is hooked to a vertex and the other end isn't hooked to anything.

We will derive a uniform bound on the position-space value of our graph, i.e. a bound which is independent of the positions of the external vertices. It is easy to use such bounds to derive bounds that are uniform in momentum space. If $k_{v}$ is the (fixed) external momentum which is entering the vertex $v$, the value of $G$ in 
momentum space is

$$
\tilde{G}\left(k_{1}, \ldots, k_{n}\right)=\int \prod_{v}\left(d^{d} x_{v} e^{i k_{v} \cdot x_{v}}\right) G\left(x_{1}, \ldots, x_{n}\right),
$$

and is bounded in absolute value by

$$
(2 \pi)^{d} \delta\left(\sum_{v} k_{v}\right) \int d^{d} x_{2} \ldots d^{d} x_{n}\left|G\left(0, x_{2}, \ldots, x_{n}\right)\right|,
$$

thanks to the translation invariance of $G\left(x_{1}, \ldots, x_{n}\right)$. Hence we need only fix the location of one external vertex at 0 and re-designate all the remaining vertices to be internal vertices.

We assume

\section{(H3.1) The Lines}

The $l^{\text {th }}$ line has a propagator which, in space-time, is bounded by $\frac{K^{\prime}}{1+|x-y|^{d-2 p(l)}}$, where $0<p(l)<\frac{d}{2}$ and $d$ is the dimension of space-time. This is the behaviour, for example, of the propagator $\frac{f(k)}{k^{2 p(l)}}$, where the ultraviolet cutoff $f(k)$ is integrable and has at least $(d+1)$ integrable derivatives.

\section{(H3.2) $\equiv(H 2.2)$ The Vertices}

Any strictly positive number of lines may be hooked to any vertex.

\section{(H3.3) The External Vertices}

There must be one or more vertices designated as external vertices. These vertices are located at fixed but arbitrary points of space-time.

The final, but, of course, principal, hypothesis is that of superficial convergence. The test for superficial convergence [LZ] is constructed as follows. Let $G^{\prime}$ be any subgraph of $G$. Let $\mathscr{C}\left(G^{\prime}\right)=\left\{H_{1}, \ldots, H_{n}\right\}$ denote the connected components of $G^{\prime}$, $\mathscr{C}_{I}\left(G^{\prime}\right)=\left\{H_{i} \in \mathscr{C}\left(G^{\prime}\right) \mid H_{i}\right.$ does not contain an external vertex of $\left.G\right\}$ denote the "internal components of $G^{\prime}$ " and $C_{I}\left(G^{\prime}\right) \equiv\left|\mathscr{C}_{I}\left(G^{\prime}\right)\right|$. You are to think of the position of all vertices within any single component of $G^{\prime}$ as being fixed relative to each other. Each internal component is free to move as a unit over all $\mathbb{R}^{d}$. The internal vertices of $G$ which do not appear in $G^{\prime}$ are free to roam independently. For example in

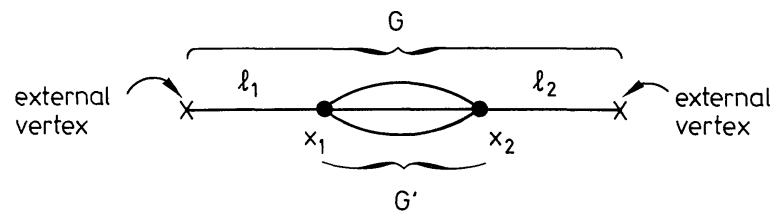

the variable $x_{1}-x_{2}$ is to be held fixed while the variable $y=\frac{x_{1}+x_{2}}{2}$ is to be integrated. The resulting integral will have power counting favorable for 
convergence if

$$
D^{I}\left(G, G^{\prime}\right)=\sum_{l \in G \backslash G^{\prime}}(d-2 p(l))-d C_{I}\left(G^{\prime}\right)-d V_{I}\left(G \backslash G^{\prime}\right)
$$

is strictly positive. Here $V_{I}\left(G \backslash G^{\prime}\right)$ is the number of vertices that are internal to $G$ but do not appear in $G^{\prime}$. The example above fails this test (barely) when $d=4$ and $p\left(l_{1}\right)$ $=p\left(l_{2}\right)=1$. In order to get a bound of the form $K^{L(G)}$ we demand further that $D^{I}\left(G, G^{\prime}\right)$ grow linearly in $E\left(G^{\prime}\right)$, the number of external lines of $G^{\prime}$. An external line of $G^{\prime}$ is a line of $G \backslash G^{\prime}$ having at least one end hooked to a vertex of $G^{\prime}$.

\section{(H3.4) Superficial Convergence}

We assume that there is an $\varepsilon>0$ such that for any $G^{\prime} \varsubsetneqq G$

$$
D^{I}\left(G, G^{\prime}\right) \geqq\left\{\begin{array}{lll}
3 \varepsilon E\left(G^{\prime}\right) & \text { if } & G^{\prime} \neq \emptyset, \\
\varepsilon & \text { if } & G^{\prime}=\emptyset .
\end{array}\right.
$$

To get a feel for the linear growth condition let us consider a massless ultraviolet cutoff $\Phi_{4}^{4}$ model. Let us also aim for bounds that are uniform in momentum space, i.e. ensure that a connected graph always has one external vertex. Then for any connected graph

$$
\begin{aligned}
D^{I}\left(G^{\prime}\right) & \equiv D^{I}\left(G^{\prime}, \emptyset\right)=\sum_{l \in G^{\prime}}(d-2 p(l))-d V_{I}\left(G^{\prime}\right) \\
& =2 L\left(G^{\prime}\right)-4\left(V\left(G^{\prime}\right)-1\right) \\
& =4-E\left(G^{\prime}\right),
\end{aligned}
$$

where now the number $E\left(G^{\prime}\right)$ of external lines of $G^{\prime}$ is determined by the condition that all vertices be hooked to exactly four lines. $D^{I}\left(G, G^{\prime}\right)$ may now be calculated

$$
\begin{aligned}
D^{I}\left(G, G^{\prime}\right) & =D^{I}(G)-\sum_{H \in \mathscr{C}\left(G^{\prime}\right)} D^{I}(H) \\
& \geqq 1+\sum_{H \in \mathscr{\mathscr { C }}\left(G^{\prime}\right)}(E(H)-4),
\end{aligned}
$$

since $D^{I}(G) \geqq 1$ by the assumed convergence of $G$. Now there cannot be any $H$ with $E(H)=0$ since $G$ is connected and there cannot be any $H$ with $E(H)=2$ because choosing $G^{\prime \prime}=G \backslash\{$ the external lines of $H\}$ results in $D^{I}\left(G, G^{\prime \prime}\right) \leqq 0$. Consequently, (H3.4) will be obeyed provided there are only a bounded number of $H$ 's with $E(H)=4$. The $\varepsilon$ that appears in $(\mathrm{H} 3.4)$ will depend on this bounded number. This is not an artificial effect. The presence of $E(H)=4$ subgraphs does indeed result in a factorial growth in the value of $G$. Consider for example

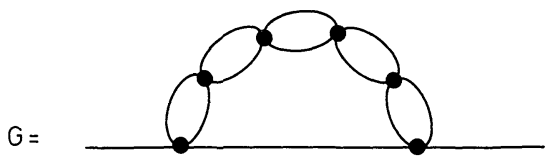

with $n$ bubbles. Now in momentum space

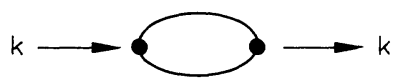


is always positive [assuming the ultraviolet cutoff $f(k)>0$ ] and is bounded below by const $\left|\ln k^{2}\right|$ for small $k^{2}$. Hence at zero external momentum

$$
|G| \geqq \text { const }^{n} \int_{|k| \geqq 1} d^{4} k\left|\ln k^{2}\right|^{n} \frac{1}{k^{2}} \geqq \text { const }^{n} n ! .
$$

Theorem 3.1. If a connected graph $G$, having at least one external vertex, obeys (H3.1)-(H3.4) above, then there exists a constant $K$, depending only on $K^{\prime}, d, \varepsilon$, and the $p(l)$ 's such that

$$
|G| \leqq K^{L(G)} .
$$

Proof. The proof is, of course, very similar to that of Theorem 2.1. It is left as an exercise. The solution to this exercise is available on request from the authors.

Remark. Theorem 3.1 overlaps with some results of [dCPR].

\section{Appendix. Graphs with Logarithmic Propagators}

In this section we extend the bounds of Sect. II to cover also completely convergent graphs whose propagators may contain logarithms of the momentum. Therefore, we bound convergent graphs in the sense of Weinberg's theorem [W], where also the case of logarithmic convergence was studied. Another motivation for this appendix comes from the study of asymptotically free theories. In these theories it is possible to do a partial resummation of the renormalized perturbation series which results in extra logarithms being introduced into some dressed vertices or propagators [GN 1 , 'tH, R]. If one uses a minimal resummation prescription (resummation of parquet graphs) this extra logarithmic convergence plays a crucial rôle in the estimates of dressed graphs [R].

We will consider connected Euclidean Feynman graphs obeying:

\section{(HA.1) The Lines}

The $l^{\text {th }}$ line has a propagator which in position space is bounded by

$$
K^{\prime} \int \frac{e^{i k \cdot(x-y)}}{\left(k^{2}+m^{2}\right)^{p(l)}\left[\ln \left(k^{2}+m^{2}+1\right)\right]^{\pi(l)}} d^{d} k
$$

with $m>0$ independent of $l$ and $p(l), \pi(l) \geqq 0$ (not both zero).

\section{(HA.2) The Vertices}

Any number of lines may be hooked to any vertex.

\section{(HA.3) The External Vertices}

We tend to view $G$ in position space; we do not view its external lines as part of $G$ but instead consider those vertices of $G$ that are ends of external lines as external vertices smeared with test functions. These test functions may be characteristic functions of standard unit cubes in position space [case (HA.3a)], general test functions of which one is in $L_{1}$ and the rest in $L_{\infty}$ [case (HA.3b)] or exponentials $e^{i k_{v} \cdot x_{v}}$ which fix the external momentum entering $v$ [case (HA.3c)]. In the last case we also assume that the propagators of (HA.1) are translation invariant. 


\section{(HA.4) Superficial Convergence}

We define for any connected subgraph $G^{\prime} \subseteq G$ a logarithmic superficial degree of divergence

$$
\Delta\left(G^{\prime}\right) \equiv \Lambda\left(G^{\prime}\right)-\sum_{l \in G^{\prime}} \pi(l), \text { where } \Lambda\left(G^{\prime}\right)=\# \text { loops in } G^{\prime}
$$

analogous to the (power) superficial degree of divergence $D\left(G^{\prime}\right)$. We assume that there is an $\varepsilon>0$ such that for every connected $G^{\prime} \cong G$ either

or

$$
\begin{gathered}
D\left(G^{\prime}\right) \geqq 2 \varepsilon E\left(G^{\prime}\right) \\
D\left(G^{\prime}\right)=0 \text { and }-\Delta\left(G^{\prime}\right) \geqq \varepsilon .
\end{gathered}
$$

In (A.2a) each external vertex of $G$ counts as having one (globally) external line.

\section{(HA.5) $D=0$ Forests}

There is a constant $C$ such that the number of proper $D=0$ forests of $G$ (i.e. forests consisting of $D=0$, one particle irreducible subgraphs of $G$ ) is bounded by $C^{L(G)}$. There is a constant $C^{\prime}$ such that for every connected $G^{\prime} \subseteq G$ with $D\left(G^{\prime}\right)=0, G^{\prime}$ has at most $C^{\prime}$ external vertices. Forests are sets of non-overlapping subgraphs (see e.g. $[\mathrm{dCR}])$.

Hypothesis (HA.5) is satisfied with $C=8, C^{\prime}=6$ in the $\phi_{4}^{4}$ model by [dCR, Lemma A.2] and by similar arguments for the Gross-Neveu ${ }_{2}$ model the vertex being either

Jor, where $\sim$ is an ultralocal boson field and the $\phi_{3}^{6}$ model.

Theorem A.1. If hypotheses (HA.1)-(HA.5) above are satisfied then for any $0<\zeta<1$ there exists a constant $K$ such that

$$
|G| \leqq K^{L(G)} \times \begin{cases}\sup _{x_{v}} \prod_{l \in G} e^{-m(1-\zeta)\left|x_{l}-y_{l}\right|} & \text { for (HA.3a) } \\ \inf _{v \in V_{E}}\left\|f_{v}\right\|_{L^{1}} \prod_{v \neq w \in V_{E}}\left\|f_{w}\right\|_{L^{\infty}} & \text { for (HA.3b) } \\ \delta\left(\sum_{v \in V_{E}} k_{v}\right) & \text { for (HA.3c) }\end{cases}
$$

Here the sup runs over all possible positions of vertices of $G$ but with the external vertices in the cubes of ( $H A .3 a$ ). The constant $K$ depends only on $m, \varepsilon, \zeta, d, K^{\prime}, C, C^{\prime}$, and the $p(l)$ 's and $\pi(l)$ 's.

Proof. The proof is similar to that of Theorem 2.1 so we only outline it. It suffices to consider case (HA.3a) (by translation invariance), $m=1$ (by scaling) and propagators for which one of $p(l), \pi(l)$ are zero (by factorization). We use (2.3), to bound the power law propagators and Lemma A.2 below to bound the logarithmic propagators.

Lemma A.2. For any $q, m>0,0<\zeta<1$, and $M>1$ there exists a constant $K=K(q, \zeta, m, M, d)$ such that

$$
\left|\int \frac{d^{d} k}{(2 \pi)^{d}} \frac{e^{i k \cdot(x-y)}}{\left[\ln \left(k^{2}+m^{2}+1\right)\right]^{q}}\right| \leqq K \sum_{i=0}^{\infty} M^{d \cdot i}\left[1+\ln M^{i}\right]^{-q-1} e^{-(1-\zeta / 2) m M^{i}(x-y)} .
$$


The proof of this lemma is very similar to that of Lemma 2.2. We remark that contrary to appearances we have not gained an "extra" logarithm. We shall see why later.

We next pick any fixed but arbitrary assignment of momenta scales [i.e. indices $i$ of (A.3)] to the lines of $G$ and perform the integration of the internal vertices over position space exactly as in Theorem 2.1. "The Integral over Position Space." The result is (ignoring for the time being the factors of $\left[1+\ln M^{i_{l}}\right]^{-\pi(l)-1}$ in the propagators and also some irrelevant constants)

$$
\prod_{j \geqq 1} \prod_{k} M^{D\left(G_{j}^{k}\right)}
$$

where the $G_{j}^{k, s}$ are the connected components of $G_{j}=\left\{l \mid i_{l} \geqq j\right\}$.

We can't at this stage simply perform the sum over momentum assignments because it is possible for $D\left(G_{j}^{k}\right)$ to be zero. Consequently, we fail to get exponential decay in momentum space. Let

$$
\begin{aligned}
& \mathscr{F}_{P}=\left\{\text { proper components of } G_{j}^{k} \text { s with } D\left(G_{j}^{k}\right)=0\right\} \quad\left(\mathscr{F}_{P} \text { is a forest }\right) \text {, } \\
& \mathscr{G}_{L}=\bigcup_{j, k}\left\{\{l\}\left|l \in G_{j}^{k}\right|\left\{\text { all proper components of } G_{j}^{k}\right\}\right\}, \\
& \mathscr{G}=\left\{\begin{array}{lll}
\mathscr{F}_{P} \cup \mathscr{G}_{L} & \text { if } & D(G)=0, \\
\mathscr{F}_{P} \cup \mathscr{G}_{L} \cup\{G\} & \text { if } & D(G) \neq 0 .
\end{array}\right.
\end{aligned}
$$

Thanks to (HA.5) the number of different possible $\mathscr{G}$ 's is bounded by $(2 C)^{L(G)}$ so it suffices to fix $\mathscr{G}$ and consider only momentum assignments giving that $\mathscr{G}$. For each $g \in \mathscr{G}$ define the base momentum

$$
b(g)= \begin{cases}0 & \text { if } g=G, \quad D(G) \neq 0, \\ \min \left\{i_{l} \mid l \in g\right\} & \text { otherwise. }\end{cases}
$$

We claim that if the $b(g)$ 's are held fixed the remaining part of the sum over momentum assignments may be done using (A.4). The idea is as follows. Fix any $g \in \mathscr{G}$ and consider $g_{r}=g / \bigcup_{\mathscr{G} \rightarrow g^{\prime} \varsubsetneqq g} g^{\prime}$ (i.e. contract all elements of $\mathscr{G}$ inside $g$ to points). Because all the "dangerous subgraphs" (i.e. $G_{j}^{k}$ s with $D=0$ ) inside $g$ have been contracted to points, (A.4) does give exponential decay between the momentum scales of internal lines of $g_{r}$ meeting at a common vertex of $g_{r}$. This calculation is done in detail in [FMRS 3, Sect. III] for the case where one need not worry about taking proper components. Having to worry about proper components is no more than an additional nuisance factor. There is no exponential decay to external lines of $g_{r}$ since $D(g)=0$, but the fact that $b(g)$ is fixed breaks translation invariance and we are still able to sum over the momentum assignments to lines of $g_{r}$. Every line of $g$ appears in precisely one $g_{r}$.

That leaves only the sum over the $b(g)$ 's. That's where the logs come in. Since

$$
\sum_{i>j}\left[1+\ln M^{i}\right]^{-\lambda} \leqq \sum_{i>j} \frac{1}{(i+1)^{\lambda}} \leqq \frac{1}{\varepsilon} \frac{1}{(j+1)^{\lambda-1}} \quad \text { if } \quad \lambda \geqq 1+\varepsilon,
$$

each sum over a $b(g)$ (think $i$ ) uses up one log from within $g$ and transmits the rest to the next larger element of $\mathscr{G}$ (think $j$ ). Hence it suffices to verify that every $g \in \mathscr{G}$ 
contains enough logarithms. The number of logs that $g$ has access to (i.e. the number in propagators of $g$ minus the number already used up in sums) is

$$
\begin{aligned}
\lambda(g) & =\sum_{l \in g}^{L}(1+\pi(l))-\operatorname{card}\left\{g^{\prime} \in \mathscr{G} \mid g^{\prime} \nsubseteq g\right\} \\
& \geqq \sum_{l \in g} \pi(l)-\operatorname{card}\left\{g^{\prime} \in \mathscr{F}_{P} \mid g^{\prime} \nsubseteq g\right\} \\
& =1+\sum_{l \in g} \pi(l)-\operatorname{card}\left\{g^{\prime} \in \mathscr{F}_{P} \mid g^{\prime} \subseteq g\right\},
\end{aligned}
$$

where $\sum^{L}$ denotes the sum over logarithmic lines. Note that the "extra" logarithms that Lemma A. 2 appeared to give us have been used up in performing the sum over the base momenta of the singleton logarithmic lines in $\mathscr{G}_{L}$. The claim that $\lambda(g)$ $\geqq 1+\varepsilon$ now follows from hypothesis (HA.4) and

Lemma A.3. $g \in \mathscr{F}_{P} \Rightarrow \operatorname{card}\left\{g^{\prime} \in \mathscr{F}_{P} \mid g^{\prime} \leqq g\right\} \leqq \Lambda(g)$.

This lemma follows by induction from the fact that any $1 P I g$ has a loop that is not contained in any disjoint union of $1 P I$ subgraphs of $g$.

\section{References}

[dCPR] de Calan, C., Petritis, D., Rivasseau, V.: In preparation

[dCR] de Calan, C., Rivasseau, V.: Commun. Math. Phys. 82, 69 (1981)

[FMRS 1] Feldman, J., Magnen, J., Rivasseau, V., Sénéor, R.: The infrared behaviour of $\phi_{4}^{4}$ (in preparation)

[FMRS 2] Feldman, J., Magnen, J., Rivasseau, V., Sénéor, R.: Méthodes pour la théorie constructive des champs renormalisables, asymptotiquement libres. Proceedings of RCP 25, Strasbourg, June 1984

[FMRS 3] Feldman, J., Magnen, J., Rivasseau, V., Sénéor, R.: Bounds on renormalized Feynman graphs. Preprint CPTh, Ecole Polytechnique

[G 1] Glimm, J.: Commun. Math. Phys. 10, 1 (1968)

[G 2] Gallavotti, G.: Renormalization theory and ultraviolet stability for scalar fields via renormalization group methods. Preprint Università di Roma II, August 1984

[GJ] Glimm, J. Jaffe, A.: Fortschritte der Physik 21, 327-376 (1973)

[GN] Gallavotti, G., Nicolo, F.: Renormalization theory in four dimensional scalar fields, Università di Roma "La Sapienza" (preprint). Commun. Math. Phys. (to appear)

['tH] 't Hooft, G.: Commun. Math. Phys. 86, 449 (1982)

[L] Lautrup, B.: On high order estimates in QED. Phys. Lett. 69 B, 109-111 (1977)

[LZ] Lowenstein, J.H., Zimmermann, W.: Commun. Math. Phys. 44, 73 (1975)

[R] Rivasseau, V.: Construction and Borel summability of planar 4-dimensional Euclidean field theory. Commun. Math. Phys. 95, 445-486 (1984)

[RS] Rivasseau, V., Speer, E.: Commun. Math. Phys. 72, 293 (1980)

[W] Weinberg, S.: Phys. Rev. 118, No. 3 (1960)

Communicated by J. Lascoux

Received September 20, 1984 